Uncovering the Anatomy of Search Without Technology

Dirk U. Wulffa,b and Ralph Hertwig ${ }^{\mathrm{b}}$

University of Basel ${ }^{\mathrm{a}}$

Max Planck Institute for Human Development ${ }^{\mathrm{b}}$

To appear as:

Wulff, D. U., \& Hertwig, R. (2018). Uncovering the anatomy of search without technology. In M. Schulte- Mecklenbeck, A. Kuehberger, \& J. G. Johnson (Ed.), A handbook of process tracing methods (2. ed.). Psychology Press.

Author note:

Corresponding Author: Dirk U. Wulff, University of Basel, Missionsstrasse 64A, 4055 Basel, dirk.wulff@gmail.com

We gratefully acknowledge editorial support from Susannah Goss and the editors' helpful comments. 


\section{Uncovering the Anatomy of Search Without Technology}

Admittedly, our chapter is a somewhat odd addition in a Handbook of Process-Tracing Methods. We will not revel at the ever growing and ever more sophisticated methods to trace processes nor will we conceive of still another technology. Nevertheless, our concern will be with "information search prior to choice" — the object of desire of what Schulte-Mecklenbeck et al. (2017) called "movement-based (process tracing) such as computer-based information boards, eye tracking, joystick and slider bar, mouse tracking or the tracking of reaching pointing” (p. 443). Our starting point is this observation: Process-tracing methods often focus on cognitive tasks that in themselves are devoid of an explicit and extended episode of search, thus requiring sophisticated technologies and efforts to look through this dimly lit and small window of search. Our chapter will be concerned with a very different way, requiring much less engineering, of laying bare the anatomy of the search process, and in fact, giving search a leading role in behavioral experiments and theories of choice. The cognitive task that we focus on is risky choice, still one of the most important domains for studying the way humans make decisions. Since the early 2000s research on risky choice has (again) begun to study experiential-based paradigms (for reviews see Hertwig \& Erev, 2009; Wulff, Mergenthaler Canseco, \& Hertwig, 2018) — often in parallel with description-based paradigms. In the experiential paradigms, the search process and its major defining properties - that is, for instance, amount of search and the search policy — unfolds for everyone to see. Experiential paradigms in risky choice and many other lines of research, according to our key argument, represent an alternative to the use of sophisticated process-tracing technologies and auxiliary assumptions necessary to lift the veil and to understand the processes preceding choice. 
Let us emphasize that our goal in this chapter is not to play process-tracing technologies against experiential paradigms. Barely any aspect of modern life-from technology, science, commerce, literary arts to news media and the World Wide Web-is conceivable without the reading and writing of symbolic descriptions. Therefore, it is important to understand how people search information in environments in which symbolic descriptions of information can be directly perused. Yet, research employing process-tracing technologies may nevertheless benefit greatly from the consideration experiential of paradigms, for instance in parallel to the description-based paradigms, thus enjoying a view on search that is less obscured and requires fewer assumptions.

\section{Two Modes of Learning and The Description-Experience Gap}

One of the most frequently studied "fruit flies" in cognitive psychology is choice between monetary lotteries. In theory, this Drosophila melanogaster can be studied from many angles. In practice, however, many researchers have grown accustomed to relying on a single one (see Pleskac \& Hertwig, 2014; Weber, Shafir, \& Blais, 2004): lotteries in which the outcomes and their probabilities are explicitly stated (either numerically or visually in terms of, e.g., pie charts), and respondents thus make decisions from description (Hertwig, Barron, Weber, \& Erev, 2004). This fruit fly_-fully described lotteries — has populated both economists' and psychologists' laboratories. For instance, one of the most famous violations of expected utility theory, the Allais paradox, involves choices between explicitly stated outcomes and probabilities (Allais, 1953, p. 514). Similarly, in his informal experiment designed to illustrate ambiguity aversion, another violation of expected utility theory, Ellsberg (1961, p. 650) relied on a setting with stated outcomes and probabilities (except in the ambiguous urn, in which probabilities were left unspecified). Hardly any learning was necessary. The same was true for Kahneman and 
Tversky's (1979) numerous demonstrations of violations of expected utility. Researchers have characterized such decisions based on full description as "static" (Edwards, 1962) and noted that: "When a static decision task is used, the decision maker does not have to learn from past experience with the outcomes of previous decisions. ... This feature of the static decision task becomes a problem when generalizing results to the many day-to-day decisions that repeatedly confront individuals, since explicit information concerning outcome probabilities is frequently not available and must be learned from previous experience.” (Busemeyer, 1982, p. 176).

Indeed, in everyday life, people are rarely able to consult explicit descriptions of probabilities and outcomes. Instead, they often cannot help but rely on past or online experience - if existent — with these options, thus making decisions from experience rather than decisions from description (Hertwig, Barron, Weber, \& Erev, 2004; Wulff et al., 2018). For instance, when crossing a street a person typically cannot rely on actuarial data; that is, there is no table of descriptions available that would inform her about the probabilities and outcomes. However, the individual will have made potentially relevant experiences in other traffic environments that she can bring to bear to the present situation and, in addition or alternatively, can explore the present situation hands-on. A similar case can be made for a plethora of situations involving real-world risks (e.g., postponing the back-up on one's hard drive, having unprotected sex, experimenting with drugs). The distinction between description-and experienced-based choice has raised a number of new questions, the two most important ones being: First, to what extent do these two modes of learning about the world result in similar or systematically different choices? Second, if they do lead to different choices, can this be reflected in the existing models of choice or are new ones required? These questions have received much attention since a set of three articles in the early 2,000s demonstrated a systematic 
discrepancy between description- and experienced-based choices ${ }^{1}$ : the description-experience gap (Barron \& Erev, 2003; Hertwig et al., 2004; Weber, Shafir, \& Blais, 2004).

To study decisions from experience researchers have commonly turned the monetary lotteries with their stated outcomes and probabilities into a kind of experience generator. One particular implementation of this generator is the sampling paradigm. Individuals initially know nothing about the options but are permitted to explore the options (they neither gain nor lose any money in so doing) by sampling possible outcomes from the payoff distributions (the options) before making a choice (typically with a press of a button; see Figure 1 for an example). Each sample usually produces a single outcome drawn (with replacement) from its respective distribution. Participants also typically determine the number of outcomes they sample (the sample size); specifically, they are instructed to sample until they feel confident enough to decide which option to choose in a final draw involving real monetary payoffs. Once they terminate sampling, they indicate their preferred option. Two related paradigms are the partial- and fullfeedback paradigms. In contrast to the sampling paradigm, the feedback paradigm suspends free information search by rendering each selection of an option a consequential choice. The feedback paradigms thus require individuals to trade-off between their exploration goal (i.e., learning about the options) and their exploitation goal (i.e., maximizing their returns). In the partial-feedback paradigm, feedback is provided in each trial only for the selected option (orange box) whereas the full-feedback paradigm provides feedback also concerning the foregone payoff

${ }^{1}$ The study of experienced-based decision making is in fact much older and harks back to the beginnings of behavioral decisions science with Edwards (1956, 1961, 1962), Rappaport (1964), and Busemeyer (1982) to give but a few references. New in the early 2000s was that researchers began to systematically pit experience- and description-based decisions against each other and to characterize whether and why decisions based on these two modes of learning diverge. 
(i.e., the payoff that the person would have received, had she selected the other option; gray box). These paradigms fostered a simple but informative comparison between decisions made from experience and decisions made from description, revealing what has become known as the description-experience gap (D-E gap; Hertwig \& Erev, 2009; Wulff et al., 2018).

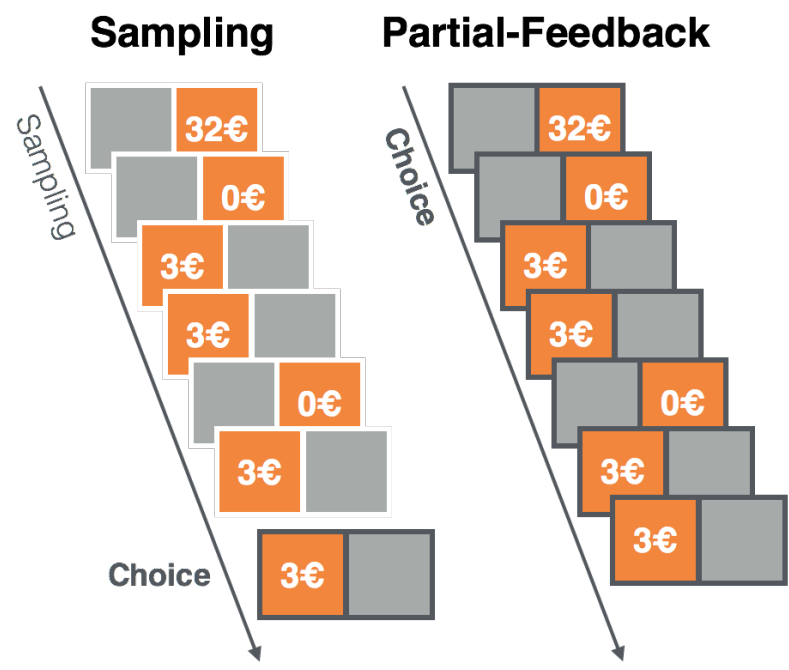

Full-Feedback

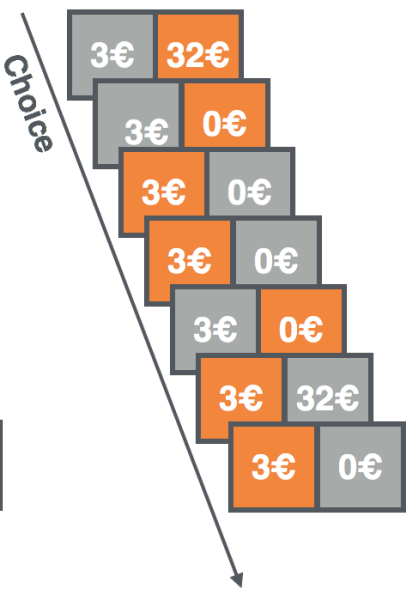

Figure 1. Paradigms of decisions from experience. See text for description.

It is now well established that decisions made from description and decisions from experience can result in systematically different choices. Recently, Wulff et al. (2018) conducted a meta-analysis of 33 datasets $^{2}$ using the sampling paradigm examining, among other analyses, the difference in the average proportion of choices consistent with Cumulative Prospect Theory ${ }^{3}$ between description and experience. When the choice problems included a risky and a safe option - the type of problem frequently used in economics and psychology to infer individuals' risk preference - the average gap size in choice behavior was large, namely, about 20 percentage

\footnotetext{
${ }^{2}$ For a description of the dataset collection see also van den Bos, Jenny, and Wulff (2014).

${ }^{3}$ Based on the parameters determined by Tversky and Kahneman (1992)
} 
points. This gap in choice behavior is, however, not premised on any particular theory of choice (e.g., one that assumes probability weighting). That is, it occurs under different measurement approaches, including approaches that evaluate choices as a function of maximization, namely whether individuals choose the option that promises the higher expected return based on the available information (see Wulff et al., 2018), or risk, where individuals choose the option with objectively higher variance (e.g., Ludvig \& Spetch, 2011). It also occurs across experimental paradigms, including the partial- and full-feedback paradigms (Figure 1; see also Hertwig \& Erev, 2009; Wulff et al., 2018), and within individuals (e.g., Camilleri \& Newell, 2009), and it represents a systematic difference from expected value maximization. Yet, the direction of this description-experience gap is typically summarized in terms of the (as-if) weighting of rare events (for a recent analysis see also Regenwetter \& Robinson, 2017). In decisions from description, individuals tend to choose as if they overweight the impact of rare events, i.e., as if rare events receive more weight than they deserve based on their objective probability. In decisions from experience, by contrast, individuals tend to choose as if they underweight rare events.

\section{The Roles of Information Search in Decisions from Experience}

Several explanations for the emergence of the description-experience gap have been proposed. In one way or another, information search is often invoked in those explanations. In the following, we address two of these explanations for the description-experience gap in the context of two different roles that information search has been found to take in decision from experience: (a) search as a driver of experience and (b) search as a signal of preference. Henceforth, we focus on the sampling paradigm of decisions from experience, as it has been the 
focus of most experimental work on the description-experience gap and its cognitive underpinnings.

\section{Search as a Driver of Experience}

Research on the sampling paradigm has shown that individuals on average rely on about 20 samples per problem, implying a sample size of about 10 samples per option (see Table 1, Wulff et al., 2018). Such frugal exploration has two important consequences for individuals' experiences. First, it will prevent many individuals from experiencing the unlikely event in the first place. For instance, when taking 10 samples from the option $\$ 4$ with probability .8 and $\$ 0$ otherwise versus $\$ 3,11 \%$ of individuals are expected to not see the unlikely event ( 0 with $20 \%$ ) at all. Second, the majority of all individuals, including those who did experience the unlikely event, are expected to see it less often than expected from its objective probability. In the above example, the expected number of times of experiencing the rare event with ten samples is two. Based on the binomial distribution, which governs the sample distribution of two outcome lotteries, $30 \%$ of individuals are expected to see the rare event exactly twice and $32 \%$ of individuals are expected to see the rare event more than twice; however, more individuals, namely $38 \%$, are expected to experience the rare event never or only once. The reason for this result is that the binomial sampling distribution is right-skewed for events with a probability smaller than .5 , implying more mass below than above the expected value, and vice versa for events with probability larger than .5. Figure 2 illustrates this regularity for 40,246 empirical sampling trials analyzed by Wulff et al. (2018): The medians of experienced relative frequencies fall below the identity line for small probabilities, tend to be clustered around the identity line for medium probabilities, and fall above the identity line for large probabilities. Furthermore, the plots of the marginal distributions in Figure 2 show that, relative to the decision problems' 
objective probabilities, the experienced relative frequencies are systematically shifted toward the boundaries (0 and 1). Based on these statistical regularities, frugal search, ceteris paribus, can explain the as-if underweighting (conditioned on the objective probabilities) regularity in decisions from experience. That is, individuals make choices that appear to underweight rare events because they tend to "under-experience" them. Thus information search has a substantial and systematic impact on the environments that individuals experience and the choices that individuals subsequently make.

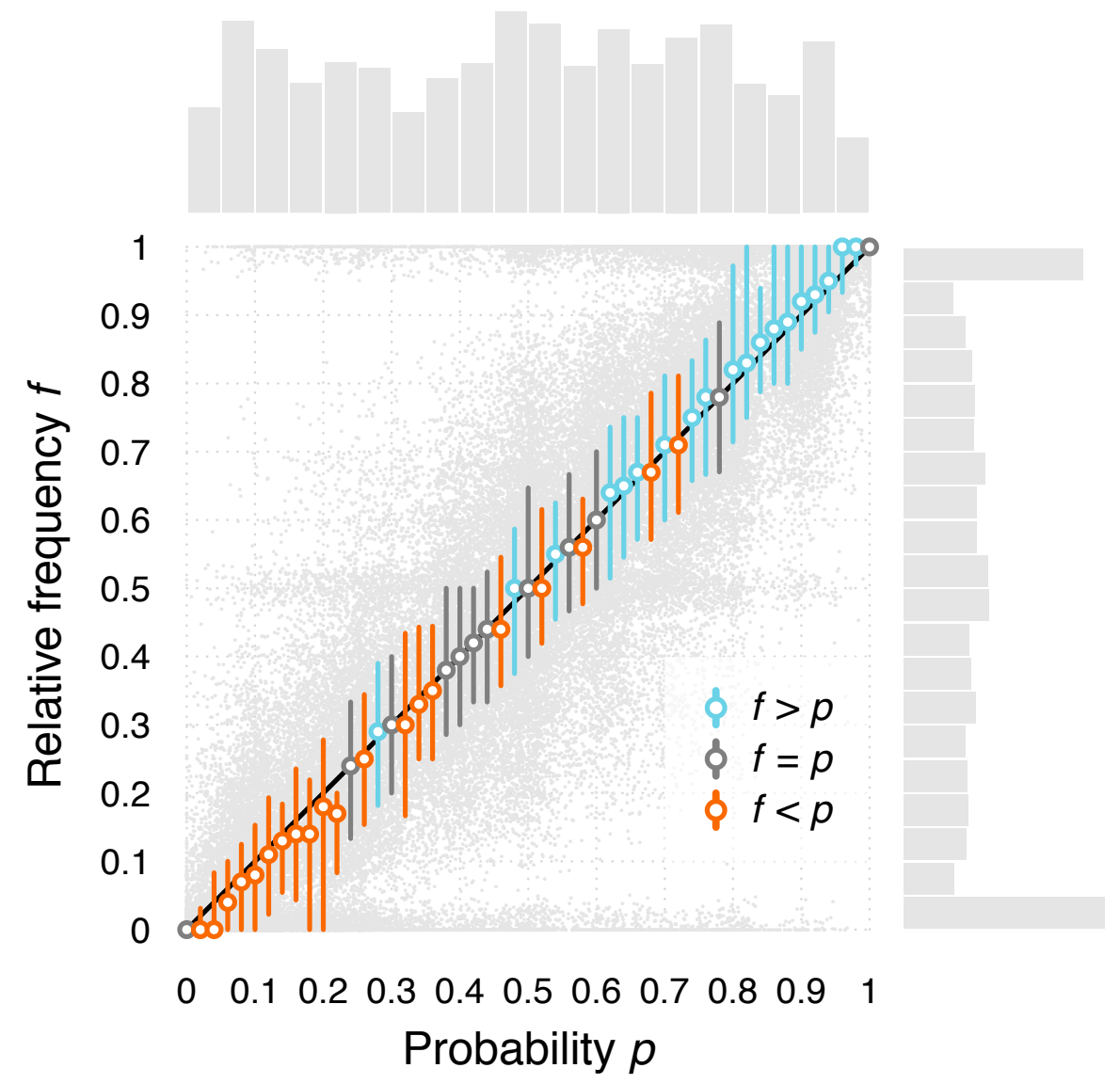

Figure 2. Small samples, sampling error, and rare events. This figure plots the true probability $\mathrm{p}$ versus the experienced relative frequency $f$ for all sampling trials in our analysis (excluding options with $p=1.0$ ). The points in the background represent the individual trials. The circles 
and lines in the foreground (in blue, gray, or orange) represent the median experienced probabilities for each unique true probability and the respective interquartile range. The bar graphs at the top and on the right show the marginal distribution of the objective probabilities and of the experienced relative frequencies, respectively. Reprint of Wulff et al. (2018).

Why do individuals curtail their search so soon and forego the opportunity to obtain an accurate representation of an option? Two factors are likely to contribute to frugal search. First, individuals usually do not know in advance the set of possible outcomes and hence cannot discern when their sample of experience offers a veridical reflection of the true state. Individuals thus cannot know for sure whether it is worth continuing to search or not. Second, the incremental value of search drastically diminishes over time. In a simulation involving risky lotteries, Hertwig and Pleskac (2010) demonstrated that although choices derived from small samples are not optimal, they are surprisingly good. With a sample as tiny as one draw, the chance of selecting the better lottery was approximately $60 \%$ in the simulated environment of 1,000 pairs of randomly generated lotteries (simulated person samples from two payoff distributions, each consisting of two outcomes of the type "a probability $\mathrm{p}$ to win amount $\mathrm{x}$; otherwise win amount y"). Drawing as few as seven times from each deck offers an $81 \%$ chance of selecting the better lottery. Moreover, accuracy continues to increase at a diminishing rate. An individual can, for example, increase the likelihood of selecting the higher expected value lottery from $60 \%$ to $78 \%$ by increasing sample size from 1 to 5 . By doubling sample size from 10 to 20 draws, accuracy increases by merely 2 percentage points. This means that beyond a small sample of draws the further investment of search effort promises increasingly small returns. In light of this cost-benefit ratio, individuals' limited exploration may be anything but unreasonable. 


\section{Search as a Signal of Preference}

Permitted to search for as long as they like, individuals usually take only a limited number of samples per payoff distribution with the consequence that the information acquired by individuals systematically misrepresents the true underlying properties and quality of the choice options. Yet, in light of the inherent uncertainty and the diminishing returns expected from continued sampling, the individual's explorative behavior represents an adaptive response to the choice environment they are in. And this is not the only indication that people adapt to internal and external characteristics of the choice environment. Wulff et al. (2018) summarized the known factors in how much people explore. For instance, individuals have been found to increase their sample size, for instance, in the presence of potential losses (Lejarraga, Hertwig, \& Gonzalez, 2012), in response to the affective state of fear (Frey, Hertwig, \& Rieskamp, 2014), and when the number of options increases (Hills, Noguchi, \& Gibbert, 2013; Noguchi \& Hills, 2015). Sampling efforts are also boosted by increasing the monetary stakes (Hau et al., 2008); similarly, Wulff, Hills, and Hertwig (2015a) showed that sample size can also be increased by incentivizing individuals to maximize the long-run rather than the short-run return. Taken together these and other results (e.g., the drastic effects of competitive versus noncompetitive search shown by Phillips et al., 2014) suggest individuals to be adaptive explorers. In their explorative behavior, they reveal their sensitivity to the costs and benefits of search and, more generally, their goals, aspirations and properties of the choice options and choice environment.

People's capacity for adaptive exploration suggests a link between search and preference, which can be utilized in order to better understand the underpinnings of choice in decisions from experience. The revealed preference approach assumes that a person's preference is laid bare through the explicit choices he or she makes. The work on experienced-based choice suggest a 
different or at least a complimentary possibility. When searching sequentially (as in the sampling paradigm) each sample offers and, in fact, requires a choice to continue or to terminate search. This implies that the number of samples that individuals draw may be another reflection of a person's preferences and vice versa. This link between search and preference has recently been conceptualized in terms of an evidence accumulation framework (Markant, Pleskac, Diederich, Pachur, \& Herwig, 2015, 2018; Wulff, Markant, Pleskac, \& Hertwig, in press; see also Ostwald, Starke, \& Hertwig, 2015; Pleskac, Yu, Hopwood, \& Liu, in press; Zeigenfuse, Pleskac, \& Liu, 2014). Specifically, the CHASE model (Choice from Accumulated Samples of Experience, 2018, in press) is built on the idea that observed outcomes contribute to an accumulated preference in proportion to their subjective value. Over the course of search, the subjective values accumulate to form a preference for one option over the other until, at some point, the preference strength reaches one of two thresholds, one for each alternative (see Figure 3). The final choice is then determined by the particular threshold that is reached and the sample size is determined by the time it takes (or, more precisely, the number of steps taken) to reach the threshold.

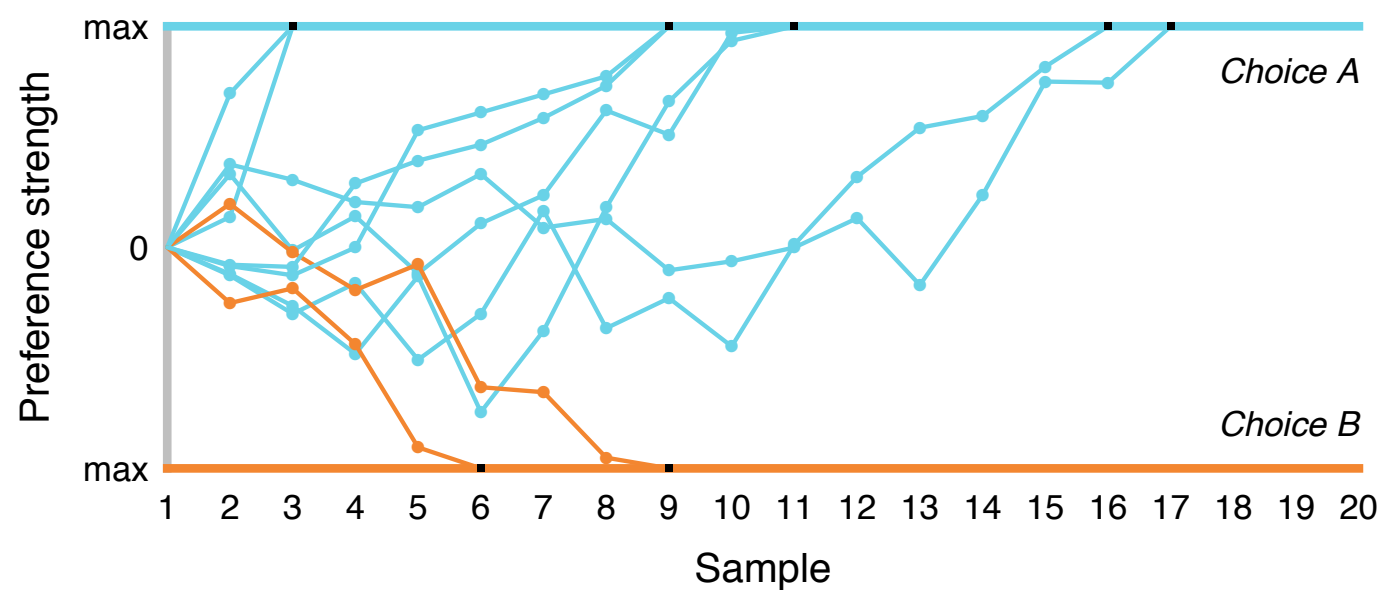

Figure 3. Model of preference accumulation. Figure illustrates for 10 hypothetical choices the accumulation of preference strength (y-axis) across samples (points) from a neutral start point 
(zero) towards one of the decision thresholds. Black squares mark the sample at which the decision threshold was reached. They thus also determine the number of samples at which point search is terminated.

Using the computational framework of CHASE, sample size can help us recover characteristics of the decision process. For instance, a lack of a clear preference in terms of choices can be the result of low threshold separation, which may be interpreted as "triggerhappy", given that relatively little evidence needs to be accumulated before a choice is made. However, the same choice behavior can also be the result of a low sensitivity to the magnitude of outcomes or probabilities, which results in only small changes of preference strengths associated with each sample. ${ }^{4}$ Thus, in this case, two independent elements of the decision process can produce the same choice behavior. What holds for choice, however, does not hold for search; that is, low threshold separation will lead to relatively small sample sizes whereas low sensitivity will lead to large sample sizes. Thus, based on a key property of information search—namely, sample size that precedes the supposed choice behavior - we can now characterize the decision process in ways we could not by considering choice alone. For the details on CHASE please see Markant et al. (2015, 2018; Wulff et al., in press).

Strong evidence for CHASE and the link between search and preference, in general, is provided by a sampling-cascade effect for exploration in the sampling paradigm analogue to the gaze-cascade effect for eye-tracking (Wulff et al., 2018). The traditional gaze-cascade effect

${ }^{4}$ CHASE weights probabilities as a function of the associated outcomes rank in the empirical cumulative distribution mimicking in the limit rank-dependent utilities models used to describe decisions from description, such as Cumulative Prospect Theory (see Markant, Pleskac, Diederich, Pachur, \& Herwig, 2015, 2018). 
refers to the finding that gaze measured through eye-tracking gradually shifts to the eventually chosen option before making a choice, a behavioral pattern that has been taken as evidence that "gaze is actively involved in preference formation" (Shimojo, Simion, Shimojo, \& Scheier, 2003, p. 1317). Similarly, individuals in the sampling paradigm have been found to also sample more often from the eventually chosen option towards the end of the sampling sequence, suggesting that sampling and, in particular the decision to terminate sampling, are closely related to the process of making a decision. Corroborating this interpretation, this sampling-cascade effect seems to only occur in self-terminated (i.e., autonomous) sampling, where the authority to stop search resides with the participant, and not in experimenter-terminated (regulated) environments, where sample size is predetermined by the experimenter (Figure 4). Notably, the gaze-cascadelike effect is a parameter-free prediction of CHASE and any evidence accumulation model.

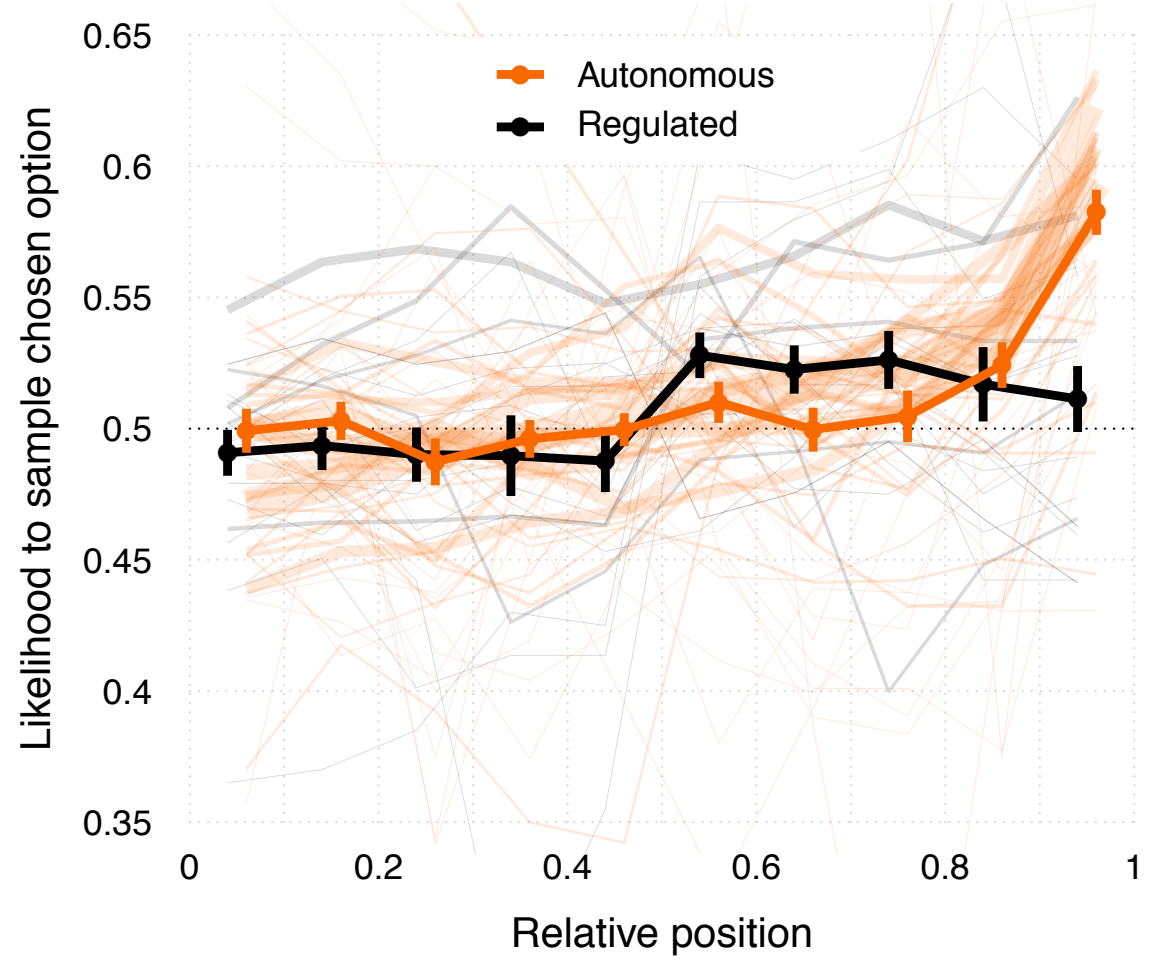


Figure 4. The sampling-cascade effect in decisions from experience. The figure shows, separately for autonomous and regulated sampling, the likelihood of sampling from the (ultimately) chosen option as a function of the draw's relative position in the sampling sequence. The lines in the background show the results separately for datasets with autonomous search termination, in which individuals self-terminate search, and datasets with regulated search termination, in which search is experimenter-terminated. The error bars represent the standard error of the mean. Reprint of Wulff et al. (2018).

An interesting consequence of the kind of optional stopping implied by CHASE and the sampling-cascade effect is that it gives rise to recency; that is, experiences sampled later in the sequence will be more correlated to the final choice than earlier ones. To see why, consider that the very last sample must necessarily be in favor of the eventually chosen option, as otherwise it would not have pushed the relative preference strength over the threshold. Similarly, the second to last sample must have likely been in favor of the eventually chosen option, as otherwise the relative preference strength would not have been close enough for the last sample to cross the threshold. And so on. This has an important consequence for the description-experience gap. Attempts to explain a description-experience gap have frequently recruited the notion of recency as the main factor leading to a gap beyond sampling error (Hertwig \& Erev, 2009; Wulff et al., 2018). Specifically, these attempts assumed that recency was the result of a decision process that assigns more weight to recent samples than earlier samples due to, for instance, memory limitations. A demonstrable result of such differential weighting is that it reduces the effective samples size and thus amplifies, on a subjective level, the "under-experiencing" of rare events (see Wulff et al., 2018). However, the finding of optional stopping, as embodied in the sampling- 
cascade effect, implies that recency may not arise from differential weighting and, thus, may actually not contribute to the description-experience gap.

Optional stopping is only one way to terminate search. Evidence suggests that individuals also employ a planned stopping strategy. For instance, when analyzing the grammar of sample sizes, we have found that multiples of 10 - for instance, 20 - occur notably more frequently as sample sizes than would be expected by chance (Spektor \& Wulff, 2018). This suggests that some individuals planned in advance to terminate search on specific prominent numbers (e.g., round numbers). Corroborating this conclusion, Markant et al. (2015) found that a plannedstopping model outperformed the online evidence-accumulation process as assumed in the CHASE model for a substantial proportion of individuals.

In sum, individuals appear to be adaptive explorers. They adjust their exploration to external and internal variables, within and across decision problems. This adaptive exploration implies that individuals engage implicitly or explicitly in some form of cost-benefit analysis that takes into account the characteristics of the environment, as well as their preferences. One way of conceptualizing the underlying process is in terms of a process of evidence accumulation. Such a process explores the two options until the accumulated preference reaches a threshold. As a result, later samples will necessarily be more indicative of choice than earlier ones, rivaling the common interpretation that recency arising from differential weighting and suggesting that factors other than recency give rise to the description-experience gap beyond sampling error. Moreover, preference will be expressed not only in choice, as assumed by the revealed preference approach, but also in observable search. In other words, the dynamics of search offer a window on the decision maker's preference. The latter conclusion converges with a similar one drawn in eye-tracking studies; see Shimojo et al., 2003). Using a computational model such as 
CHASE, information search can thus be utilized to shed light on the underlying preference in ways that would not be possible based on choice alone.

\section{Conclusions}

Traditional process-tracing techniques such as Mouselab (Chapters 6-7), eye-tracking (Chapters 1-5), or Mouse-tracking (Chapters 8-10) seek to shed light on the cognitive processes underlying behavior by making observable how individuals search for information. To achieve this, one of two paths is usually taken: One is to conceal the available information so that individuals' actions to uncover the pieces of information can be recorded, such as in the case of Mouselab and Flashlight (Schulte-Mecklenbeck, Murphy, \& Hutzler, 2011). Another is to infer information acquisition from behavioral proxies such as ocular fixations or hand movements, such as in the case of eye- and hand-tracking. Here we have proposed a third path for studying information search. It rests on "experiential" paradigms that put search center stage and readily lay bare the information-search behavior for everyone to see (i.e., public rather than private search, external rather than internal search), without the use of technological crutches. In this sense, experiential paradigms represent an alternative or, at least, complement the arsenal of existing sophisticated process-tracing methodologies. In making our case for the utility of experiential paradigms that naturally disclose search processes, we have taken advantage of recent research on the description-experience gap. Although a still relatively young line of research, it has already offered interesting insights into how search and choice appear to be coupled when people face uncertainty and incomplete information and search is the strategy to reduce both.

The sampling paradigm in research on decisions from experience and the descriptionexperience gap allowed us and other researchers to identify different ways in which search can 
foreshadow choice. In experiential paradigms, one can easily gauge the amount and content of people's encoded information. In addition, one can do so without worrying, or at least less so, about delicate distinctions such as that between the initial phase of reading, in which the experimental stimuli are encoded, and the subsequent phase of information acquisition that is taken to be indicative of specific choice strategies at work. Assumptions about this reading phase (e.g., all boxes in Mouselab are examined once) are critical for quantitative models of information acquisition and can make the tests of different choice models on this basis quite tricky (see the discussion in the Appendix B of Pachur, Hertwig, Gigerenzer, \& Brandstätter, 2013). Of course, this does not mean that the experiential paradigms and the interpretation of the collected search data would be free of assumptions such as, for instance, about the relative memorability of events (also of relevance in process-tracing technology) — but our conjecture is that the number is smaller.

We do not question that process-tracing technologies represent valuable efforts to reveal processes of information acquisition and eventually move to model search and decision processes. Indeed, recent investigations have begun to entertain cell openings or eye-fixations as inputs to computational models of choice, such as the attentional drift-diffusion model (e.g., Krajbich, Armel, \& Rangel, 2010; see als Chapters 22-24). Yet is it noteworthy - and here we take research on risk choice as rather representative of the use of process-tracing technologiesthat these technologies are often used in the context of decisions from description. Individuals in description-based studies can usually quite easily obtain complete information about the available options. In contrast, this is not possible — or is at least much more difficult—for individuals in experienced-based studies. Consequently, in decisions from description the space for information search to profoundly shape experience and knowledge and inter-individual 
differences therein is often limited to the rarer cases in which individuals fail to open or glance at a piece of information at all. This implies that the most important (but by no means only) role of information in experiential choice — namely how small samples interact with the environment to produce systematically "biased" experiences (relative to the "true" environment)—is by design excluded in traditional process-tracing studies. Admittedly, employing process tracing in the context of decisions from description, with all information accessible, can sometimes help to boost sensitivity for detecting associations between search policy and strategy use. However, this focus comes at the price of passing over key properties of the mortal decision makers, namely, uncertainty and incomplete knowledge.

Research on the description-experience gap in choice between monetary lotteries is of key importance because findings from the description-only paradigm "have formed the bedrock of contemporary decision theories, most notably prospect theory" (Fantino \& Navarro, 2012, p. 303); however, the impact of the description-experience distinction is not limited to choices between monetary lotteries. Numerous other choice and judgment phenomena have, for several decades, been studied primarily with description-based paradigms, including base-rate neglect, sunk-cost effects, and social and strategic dilemmas (see Fantino \& Navarro, 2012). Recently, researchers have begun to examine the possibility of description-experience gaps in other domains, such as temporal discounting (Dai, Pachur, Pleskac, \& Hertwig, 2018; Kemel \& Travers, 2016), strategic reasoning in social games (Fleischhut, Artinger, Olschewski, Volz, \& Hertwig, 2014; Martin, Gonzalez, Juvina, \& Lebiere, 2014), consumer choice (Wulff, Hills, \& Hertwig, 2015b), medical decisions and reasoning (Armstrong \& Spaniol, 2017; Fraenkel, Peters, Tyra, \& Oelberg, 2016), and adolescent risk taking (Pollak et al., 2016; van den Bos \& Hertwig, 2017). This means that turning to the process of exploration and learning with the help 
of experiential paradigms to understand the cognitive processes behind a wide range of important cognitive tasks has just begun.

Let us highlight again what we said at the outset. Our point is not to play process-tracing methodologies against experiential paradigms. The ability to search and explore and to learn from experience as well as the ability to process symbolic descriptions are both hallmarks of human cognition. Yet, research harnessing process-tracing methodologies has — to the best of our knowledge - exclusively focused on decisions from description. This requires sophisticated methodologies and auxiliary assumptions to interpret the data. Experiential paradigms render it easier, according to our conjecture, to study search and how it shapes choice. Let us conclude by inviting everyone to test this conjecture, for instance by employing simultaneously experiential paradigms and process-tracing technologies, and, equally important, by conceiving of ways to utilize such technologies to study how the mind navigates uncertainty. Let us also find out to what extent the findings from both windows on the processes suggest converging or diverging inferences and interpretations. In the end, we believe it is not method fetishism that fosters progress but healthy competition. 


\section{Recommended reading list}

- Wulff, Mergenthaler-Canseco, and Hertwig, (2018): Meta-analysis demonstrating a systematic discrepancy between choices based on description and experience and evaluating the various drivers involved in creating this description-experience gap.

- Wulff, Hills, and Hertwig, (2015): Experimental study establishing a link between an individual's goals and information search in decisions from experience.

- Hertwig, Hogarth, and Lejarraga, (2018): Review working out the psychological implications associated with two distinct ways, experience and descriptions, to learn about choice options. 


\section{References}

Allais, M. (1953). Le comportement de l'homme rationnel devant le risque, critique des postulates et axiomes de l'école americaine. Econometrica, 21, 503-546.

Armstrong, B., \& Spaniol, J. (2017). Experienced probabilities increase understanding of diagnostic test results in younger and older adults. Medical Decision Making, 37(6), 670679.

Barron, G., \& Erev, I. (2003). Small feedback-based decisions and their limited correspondence to description-based decisions. Journal of Behavioral Decision Making, 16(3), 215-233.

Busemeyer, J. R. (1982). Choice behavior in a sequential decision-making task. Organizational Behavior and Human Performance, 29(2), 175-207.

Camilleri, A. R., \& Newell, B. R. (2009a). The role of representation in experience-based choice. Judgment and Decision Making, 4(7), 518-529.

Dai, J., Pachur, T., Pleskac, T., \& Hertwig, R. (2018). A description-experience gap in intertemporal choice. Manuscript submitted for publication.

Edwards, W. (1962). Dynamic decision theory and probabilistic information processing. Human Factors, 4(2), 59-74.

Ellsberg, D. (1961). Risk, ambiguity, and the Savage axioms. The Quarterly Journal of Economics, 75(4), 643-669.

Fantino, E., \& Navarro, A. (2012). Description-experience gaps: Assessments in other choice paradigms. Journal of Behavioral Decision Making, 25(3), 303-314.

Fleischhut, N., Artinger, F., Olschewski, S., Volz, K. G., \& Hertwig, R. (2014). Sampling of social information: Decisions from experience in bargaining. In P. Bello, M. Guarini, M. 
McShane, \& B. Scassellati (Eds.), Program of the 36th annual conference of the cognitive science society (pp. 1048-1053). Austin, TX: Cognitive Science Society.

Fraenkel, L., Peters, E., Tyra, S., \& Oelberg, D. (2015). Shared medical decision making in lung cancer screening: Experienced versus descriptive risk formats. Medical Decision Making, $36(4), 518-525$.

Frey, R., Hertwig, R., \& Rieskamp, J. (2014). Fear shapes information acquisition in decisions from experience. Cognition, 132(1), 90-99.

Hau, R., Pleskac, T. J., Kiefer, J., \& Hertwig, R. (2008). The description-experience gap in risky choice: The role of sample size and experienced probabilities. Journal of Behavioral Decision Making, 21(5), 493-518.

Hertwig, R., Barron, G., Weber, E. U., \& Erev, I. (2004). Decisions from experience and the effect of rare events in risky choice. Psychological Science, 15(8), 534-539.

Hertwig, R., \& Erev, I. (2009). The description-experience gap in risky choice. Trends in Cognitive Sciences, 13(12), 517-523.

Hertwig, R., \& Pleskac, T. J. (2010). Decisions from experience: Why small samples? Cognition, $115(2), 225-237$.

Hills, T. T., Noguchi, T., \& Gibbert, M. (2013). Information overload or search-amplified risk? Set size and order effects on decisions from experience. Psychonomic Bulletin \& Review, 20(5), 1023-1031.

Kahneman, D., \& Tversky, A. (1979). Prospect theory: An analysis of decision under risk. Econometrica: Journal of the Econometric Society, 47(2), 263-291.

Kemel, E., \& Travers, M. (2016). Comparing attitudes toward time and toward money in experience-based decisions. Theory and Decision, 80(1), 71-100. 
Krajbich, I., Armel, C., \& Rangel, A. (2010). Visual fixations and the computation and comparison of value in simple choice. Nature Neuroscience, 13(10), 1292-1298.

Lejarraga, T., Hertwig, R., \& Gonzalez, C. (2012). How choice ecology influences search in decisions from experience. Cognition, 124(3), 334-342.

Ludvig, E. A., \& Spetch, M. L. (2011). Of black swans and tossed coins: Is the descriptionexperience gap in risky choice limited to rare events? PLoS ONE, 6(6), 20262.

Markant, D. B., Pleskac, T. J., Diederich, A., Pachur, T., \& Hertwig, R. (2015). Modeling choice and search in decisions from experience: A sequential sampling approach. In D. C. Noelle, R. Dale, A. S. Warlaumont, J. Yoshimi, T. Matlock, C. D. Jennings, \& P. P. Maglio (Eds.), Proceedings of the 37th annual meeting of the cognitive science society (pp. 1512-1517). Austin, TX: Cognitive Science Society.

Markant, D., Pleskac, T. J., Diederich, A., Pachur, T., \& Hertwig, R. (2018). Choosing from accumulated samples of experience. Manuscript in preparation.

Martin, J. M., Gonzalez, C., Juvina, I., \& Lebiere, C. (2014). A description-experience gap in social interactions: Information about interdependence and its effects on cooperation. Journal of Behavioral Decision Making, 27(4), 349-362.

Noguchi, T., \& Hills, T. T. (2016). Experience-based decisions favor riskier alternatives in large sets. Journal of Behavioral Decision Making, 29(5), 489-498.

Ostwald, D., Starke, L., \& Hertwig, R. (2015). A normative inference approach for optimal sample sizes in decisions from experience. Frontiers in Psychology, 6, 1342

Pachur, T., Hertwig, R., Gigerenzer, G., \& Brandstätter, E. (2013). Testing process predictions of models of risky choice: A quantitative model comparison approach. Frontiers in Psychology, 4, 646. 
Pleskac, T. J., \& Hertwig, R. (2014). Ecologically rational choice and the structure of the environment. Journal of Experimental Psychology: General, 143(5), 2000-2019.

Pleskac, T. J., Yu, S., Hopwood, C., \& Liu, T. (in press). Characterizing deliberation during preferential choice. Decision.

Pollak, Y., Oz, A., Neventsal, O., Rabi, O., Kitrossky, L., \& Maeir, A. (2016). Do adolescents with attention-deficit/hyperactivity disorder show risk seeking? Disentangling probabilistic decision making by equalizing the favorability of alternatives. Journal of Abnormal Psychology, 125(3), 387-398.

Shimojo, S., Simion, C., Shimojo, E., \& Scheier, C. (2003). Gaze bias both reflects and influences preference. Nature Neuroscience, 6(12), 1317-1322.

Schulte-Mecklenbeck, M., Murphy, R. O., \& Hutzler, F. (2011). Flashlight: Recording Information Acquisition Online. Computers in human behavior, 27, 1771-1782.

Schulte-Mecklenbeck, M., Johnson, J. G., Böckenholt, U., Goldstein, D. G., Russo, J. E., Sullivan, N. J., \& Willemsen, M. C. (2017). Process-tracing methods in decision making: On growing up in the 70s. Current Directions in Psychological Science, 26(5), 442-450.

Spektor, M. S., \& Wulff, D. U. (2018). Information search in decisions from experience. Manuscript in preparation.

Tversky, A., \& Kahneman, D. (1992). Advances in prospect theory: Cumulative representation of uncertainty. Journal of Risk and uncertainty, 5(4), 297-323.

van den Bos, W., \& Hertwig, R. (2017). Adolescents display distinctive tolerance to ambiguity and to uncertainty during risky decision making. Scientific Reports, 7, 40962.

van den Bos, W., Jenny, M. A., \& Wulff, D. U. (2014). Open minded psychology. In S. A. Moore (Ed.), Issues in open research data (pp. 107-127). London, UK: Ubiquity Press. 
Weber, E. U., Shafir, S., \& Blais, A. R. (2004). Predicting risk sensitivity in humans and lower animals: Risk as variance or coefficient of variation. Psychological Review, 111(2), 430445.

Wulff, D. U., Hills, T. T., \& Hertwig, R. (2015a). How short- and long-run aspirations impact search and choice in decisions from experience. Cognition, 144, 29-37.

Wulff, D. U., Hills, T. T., \& Hertwig, R. (2015b). Online product reviews and the descriptionexperience gap. Journal of Behavioral Decision Making, 28(3), 214-223.

Wulff, D. U., Mergenthaler-Canseco, M., \& Hertwig, R. (2018). A meta-analytic review of two modes of learning and the description-experience gap. Psychological Bulletin, 144(2), $140-176$.

Wulff, D. U., Markant, D. B., Pleskac, \& Hertwig, R. (in press). Adaptive exploration: What you see is up to you. In R. Hertwig, T. Pleskac, T. Pachur, \& The ARC Research Group (in press). Taming uncertainty. Cambridge, MA: MIT Press.

Zeigenfuse, M., Pleskac, T. J., \& Liu, T. (2014). Rapid decisions from experience. Cognition, 131(2), 181-194. 